\title{
ANÁLISIS FUNCIONAL DE LA FORMA LATINA FORTE ${ }^{1}$
}

\author{
José RAMÓN URÍZAR SALINAS \\ Universidad Autónoma de Madrid
}

\begin{abstract}
RESUMEN
El objetivo de este artículo es ofrecer una descripción funcional completa y coherente de la forma latina forte que simplifique el difícil panorama descriptivo de esta forma en los diccionarios y otros trabajos. Desde la información que estos ofrecen y a través de la obra $A b$ urbe condita de Tito Livio (siglos I a. C. - I d. C.) se analiza el funcionamiento de forte en los tres niveles del discurso propuestos por Kroon 1995: (i) en el «nivel representativo» se estudia su designación y comportamiento semántico-sintáctico en la oración; (ii) en el «nivel presentativo» se analiza su papel en la organización de eventos en los episodios narrativos; (iii) y en el «nivel interactivo» se observa el papel que desempeña en el intercambio comunicativo entre el hablante-escritor y el oyente-lector. A través de este enfoque se organizan los valores de forte en un esquema funcional en el que cada función responde a una determinada proyección en uno de los niveles comunicativos notados.
\end{abstract}

Palabras clave: causalidad, forte, función, latín, marcador discursivo.

\section{ABSTRACT}

The aim of this article is to offer a complete and coherent functional description of the Latin form forte in order to simplify the difficult descriptive overview as it appears in dictionaries and other works. Using the information that these works offer and Titus Livy's $A b$ urbe condita ( $1^{\text {st }}$ century B.C. $-1^{\text {st }}$ century A. D.) we analyze the function of forte on the three levels of discourse proposed by Kroon 1995: (i) on the «representational level» we study its designation and semantic and syntactic behaviour in the sentence; (ii) on the "presentational level» we analyze its role in the organization of events in narrative episodes; (iii) on the «interactional level» we observe its function in the communicative exchange between a speaker-writer and an addressee-reader. Using this perspective, we organize the uses of forte in a functional

${ }^{1}$ Este artículo nace del trabajo El adverbio latino forte: de la oración al discurso, TEA defendido por el autor el 25 de marzo de 2010 en la Universidad Autónoma de Madrid. Vaya mi agradecimiento a la Prof. M. $^{-\underline{a}}$ Esperanza Torrego Salcedo, directora del trabajo arriba mencionado, por su ayuda en la creación y redacción de este artículo, cuyos resultados, sin embargo, son exclusivamente de mi responsabilidad. Mi reconocimiento también a Beatriz Moncó por sus comentarios y sugerencias sobre la redacción y el contenido. Este trabajo se ha desarrollado en el marco del proyecto de investigación FFI2009-13402-C04-01. 
scheme, where each of its functions corresponds to a certain projection on one of the three communicative levels indicated.

Key Words: causality, forte, function, Latin, discourse marker.

\section{INTRODUCCIÓN}

El objeto de estudio de este trabajo es la función comunicativa de la forma latina forte en la obra titulada $A b$ urbe condita, compuesta en latín por el historiógrafo romano Tito Livio en época clásica (siglos I a. C. - I d. C.). Este estudio nace como respuesta a la complejidad descriptiva patente en los diccionarios y otros trabajos dedicados al análisis de esta forma. Todos ellos proponen un extenso catálogo de valores según los contextos en los que aparece y sus posibles traducciones en alguna de las lenguas actuales. El objetivo es ofrecer una descripción de forte lo más completa y coherente posible a partir de su estudio en los distintos niveles discursivos del modelo teórico de Kroon 1995.

\subsection{Estado de la cuestión}

Disponemos al menos de tres estudios previos de la forma forte: el trabajo de Champeaux 1967, el de Orlandini 1997 y la información que se recoge en los diccionarios.

\subsubsection{Champeaux 1967}

El trabajo de Champeaux 1967 atiende a las propiedades semánticas y pragmáticas de forte desde sus posibles traducciones al francés. Distingue así cinco usos de forte:

a) para expresar la improbabilidad o duda (par hazard, d'aventure);

b) para indicar que un evento es fortuito o una acción involuntaria (par hazard, au hazard, par inadvertance);

c) para presentar o introducir una situación determinada (il se trouve que...);

d) para indicar la coincidencia de dos eventos o insistir en su realidad (justement, précisément);

e) para expresar buena fortuna (par bonheur) o suerte (par chance).

Esta propuesta presenta algunos problemas: 
En primer lugar, es difuso el límite entre la improbabilidad, la duda (a) y la casualidad (b), como se observa en el uso de forte en contextos hipotéticos:

(1) Hercules [...] pergit ad proximam speluncam, si forte eo uestigia ferrent («Hércules se dirigió a la cueva más cercana, por si acaso las huellas llevaban hasta allí», Liv. 1.7.6).

En segundo lugar, no se entiende como valor distinto y autónomo la expresión de «buena fortuna» (e), cuando forte puede significar también todo lo contrario, la 'mala suerte'. Ejemplos como (2) demuestran que forte designa un «azar» general y no marcado, cuya connotación positiva (buena suerte) o negativa (mala suerte) depende del contexto:

(2) magno cum periculo suo, qui forte patrum in foro erant, in eam turbam inciderunt («Con gran peligro, los senadores que estaban casualmente [i.e. para su desgracia] en el foro fueron a caer en esta turba», Liv. 2.23.9).

En tercer lugar, no se explica que forte pueda desarrollar valores contradictorios. Llama la atención que unas veces exprese la duda del hablante-escritor (a-b, ej. 1) y, en otras, exprese la seguridad sobre la realidad de un determinado hecho (c-d, ej. 3):

(3) eodem forte quo haec gesta sunt die naues... rediere («Casualmente en este mismo día en el que sucedió esto las naves volvieron», Liv. 29.35.1).

En último lugar, no se considera la posibilidad de que forte pueda en ocasiones desempeñar simultáneamente varias funciones, como, por ejemplo, que pueda expresar la casualidad de un hecho (b) y, al mismo tiempo, pueda servir como eje introductor de un evento en la narración (c), situación que se da con bastante frecuencia en Livio. En (4) los saqueos recíprocos y a la vez casuales entre albanos y romanos introducen un nuevo episodio narrativo, la guerra entre ambas naciones:

(4) forte euenit ut agrestes Romani ex Albano agro, Albani ex Romano praedas in uicem agerent («Casualmente sucedió que los campesinos romanos saquearon el campo albano y, viceversa, los albanos el campo romano», Liv. 1.22.3).

\subsubsection{Orlandini 1997}

El trabajo de Orlandini 1997 considera la integración sintáctica de forte en la predicación, da cuenta también de su semántica y lo relaciona con otras formas equivalentes del italiano (per caso) y francés (par hazard). Distingue así tres tipos de forte: forte ${ }^{1}$, que actúa como adverbe de constituant 'adverbio de constituyente', expresando la casualidad de un hecho; forte ${ }^{2}$, como adverbe d'énoncé 'adverbio de enunciado', en es- 
tructuras hipotéticas, y forte $e^{3}$, como adverbe d'énonciation 'adverbio de enunciación', de expresión de duda.

Orlandini ofrece, por tanto, una primera aproximación sintáctica para forte. La descripción semántica y pragmática resulta, sin embargo, más limitada que la de Champeaux, quedándose sin mención ni explicación algunos valores determinantes como, por ejemplo, su papel en la organización de los contenidos narrativos, un uso muy abundante en la historiografía de Livio.

\subsubsection{Diccionarios}

Los diccionarios proponen, en general, unos sentidos que coinciden con los que presentan en Champeaux y Orlandini. Sucede, sin embargo, que, puesto que su finalidad última es principalmente la ayuda en la traducción, incluyen un extenso elenco de posibilidades contextuales que complican la descripción de forte, notando aquellos casos en los que la forma aparece junto a otros elementos con los que parece conformar una unidad funcional, como es el caso de ne forte, si forte, tum forte, forte temere, etcétera.

\subsection{Objetivos}

Las propuestas de Champeaux, de Orlandini y de los diccionarios son, como hemos visto, dispares tanto en la cantidad como en la calidad de la información que recogen. Este complejo horizonte descriptivo hace necesario, por tanto, revisar el uso de forte y establecer un cuadro funcional que reúna y explique de manera conjunta y coherente sus distintos valores, identificando la función o funciones más originales, a partir de las cuales se puedan interpretar otros usos derivados o contextuales. Además, se pretende también dar respuesta a dos cuestiones problemáticas:

(i) La primera es la tesis de que forte es vehículo de expresión de la duda del hablante, interpretación que nace de comparar esta forma con otros adverbios epistémicos de expresión de probabilidad con los que se relaciona etimológicamente, como forsitan y fortasse ${ }^{2}$. La realidad es que, como se verá más adelante $(\$ 4.2)$, el vínculo existente es más etimológico que funcional.

\footnotetext{
${ }^{2}$ Hand 1969 indica que forsitan proviene de la locución fors sit an (p. 713, vol. 2) y fortasse de forte an sit o forte an si uis (p. 720, vol. 2). Otros adverbios relacionados semántica y etimológicamente con forte son forsit, forsan, fortassis, fortuito y fortuitu. Sobre la expresión de duda a través de fortasse y forsitan, cf. Urízar 2010.
} 
(ii) La segunda cuestión tiene que ver con el papel de esta forma en la organización de contenidos narrativos, valor que, aunque ya ha sido notado, no ha sido explicado suficientemente ${ }^{3}$. La abundancia de este uso en la literatura latina, especialmente en obras narrativas y, dentro de este género, en Livio, justifica el análisis detenido de esta función.

\subsection{Base teórica y metodología}

Partimos del presupuesto teórico de que el acto comunicativo es un fenómeno complejo, aprehensible a través de varios niveles de estudio que se superponen y complementan. Según esta idea, cada uno de los valores de forte responde a una distinta actuación de este elemento en un determinado nivel comunicativo, lo que explica que esta forma pueda, en muchos casos, desempeñar varias funciones simultáneamente desde distintos planos de actuación.

De entre todas las propuestas teóricas que aceptan este postulado seguiremos la división tripartita del discurso de Kroon 1995, trabajo dedicado al análisis de algunas partículas latinas. Consideramos esta elección metodológicamente adecuada para el tratamiento de forte no solo porque haya sido ya aplicada al latín, sino también porque la forma presenta algunos rasgos semejantes a los de algunas partículas tratadas a través de este modelo de análisis ${ }^{4}$.

Los tres «niveles del discurso» (levels of discourse) que postula Kroon 1995, reciben el nombre de «nivel representativo», «nivel presentativo» y «nivel interactivo», cada uno de ellos referido a una dimensión distinta del acto comunicativo:

(i) El «nivel representativo» se refiere al estudio del mundo referencial (real o imaginario) que se representa a través de la lengua, esto es, el universo formado por las entidades y propiedades que configuran los eventos y las ideas ${ }^{5}$. En nuestro

\footnotetext{
${ }^{3}$ Hand 1969, vol. 2, p. 734: denique ad rerum cohaerentiam refertur. Nam forte fit id, quod in aliquod certum tempus cadit, aut cum alia re evenit una «En último lugar se refiere a la coherencia de los contenidos. En efecto, se produce por casualidad aquello que en un determinado momento acaece o coincide con otro evento". Hand ofrece, entre otros, el siguiente caso de forte con orientación textual: erat forte brumae tempus (Liv. 21.54.7).

${ }^{4}$ Un rasgo que forte comparte con las partículas de Kroon es su posición inicial en la estructura oracional, a veces en posición tmética (como autem, vero y tamen) y su valor en la organización de contenidos.

${ }^{5}$ Kroon 1995, p. 59: «A first function of coherent language is to portray or represent some real or imaginary world outside the language itself, and often, but not necessarily, also outside the immediate discourse situation».
} 
corpus corresponde con los contenidos propiamente narrativos: los personajes, los lugares, la acción, el tiempo, etc. Responde, en definitiva, a los hechos históricos (res gesta) que Livio transmite a su auditorio.

(ii) $\mathrm{El}$ «nivel presentativo», por otro lado, tiene que ver con la manera en la que se organizan los contenidos representados ${ }^{6}$. Así, el hablante puede elegir presentar los hechos respetando la secuencia lógica temporal, desde el principio hasta el final, o bien puede introducir saltos y digresiones. En el «nivel presentativo» el autor, por tanto, elige de qué manera introduce los contenidos en vista a que el acto comunicativo -en este caso informativo- sea eficaz.

(iii) En último lugar, el «interactivo» es el nivel del propio acto comunicativo, en el que el hablante-escritor, en este caso Livio, transmite su mensaje-texto al oyente-lector ${ }^{7}$. Aquí se considera todo lo que respecta a la interacción entre los participantes del acto comunicativo ${ }^{8}$.

El estudio de forte a través de estos tres niveles se plantea de la siguiente manera: por una parte, en el «nivel representativo» interesa su designación y la función semántico-sintáctica que desarrolla en la oración en la que se integra; en segundo lugar, en el «nivel presentativo» se atiende a su utilidad en la disposición de los eventos en la línea narrativa; y, por último, en el «nivel interactivo» se observa su papel en el propio acto comunicativo.

\subsection{Corpus}

El corpus empleado incluye las citas de los diccionarios y de los trabajos de Champeaux y Orlandini. Hemos querido añadir al conjunto

${ }^{6}$ Kroon 1995, p. 61: «The presentational level [...] is concerned not with the expression (i.e. the linguistic form) of the information, but rather with the presentation and organization of the information. The presentational level captures the fact that a language user imposes an organizing and rhetorical perspective on the ideas conveyed».

${ }^{7}$ Kroon 1995, p. 89: «The interactional level of discourse [...] is concerned with the coherence or incoherence of a unit of discourse in the light of the conversational exchange taking place between two or more interlocutors within a particular communicative situation».

${ }^{8}$ La relación entre los niveles del discurso de Kroon 1995 y los elementos que constituyen una oración se explica en Torrego y Villa 2009, p. 55: «El nivel mínimo (de la estructura predicativa) es la predicación nuclear; el siguiente la predicación expandida; juntos configuran el nivel representativo, en el que se expresa la descripción del evento o situación a que hace referencia el enunciado. Además, la oración puede contener información referente a la propia forma de presentar el enunciado a los participantes en el acto de habla; estos contenidos constituyen los niveles presentativo e interactivo de la oración». 
la totalidad de casos de forte encontrados en Ab urbe condita de Tito Livio (59 a. C. -17 d. C. $)^{9}$, de manera que se pueda observar el uso -muy productivo- de esta forma en un solo autor. El siguiente cuadro muestra la variedad de los casos encontrados:

\begin{tabular}{|l|l|c|}
\hline \multicolumn{2}{|l|}{ cláusula principal } & 92 \\
\hline \multirow{5}{*}{ cláusula subordinada } & participial & 28 \\
\cline { 2 - 3 } & condicional (si, nisi) & 23 \\
\cline { 2 - 3 } & circunstancial $^{10}$ (cum narrativo-histórico) $^{-}$ & 17 \\
\cline { 2 - 3 } & relativa (qui) & 15 \\
\cline { 2 - 3 } & completiva (AcInf, quod, ne) & 9 \\
\cline { 2 - 3 } & causal (quia, quod, quoniam) & 7 \\
\cline { 2 - 3 } & final (ut, ne) & 1 \\
\cline { 2 - 3 } & modal (uelut) & 195 \\
\hline
\end{tabular}

CuAdro 1: Distribución de los casos de forte en Tito Livio

Para comprobar y confirmar algunos usos y contextos de forte hemos empleado también el motor de búsqueda de la base de datos BTL2, donde se dispone de un completo catálogo de autores latinos de época clásica.

\subsection{Estructura del artículo}

El artículo se estructura según los tres niveles de estudio de Kroon 1995. El epígrafe $\$ 2$ se dedica al estudio de forte en el «nivel representativo», donde interesa su designación (\$2.1) y, en el ámbito de la oración, sus características sintácticas $(\$ 2.2)$ y semánticas $(\$ 2.3)$. En $§ 3$ se estudia su papel en el «nivel presentativo»: primero se observan las ca-

${ }^{9}$ De los 142 libros originales de $A b$ urbe condita conservamos tan solo 35: los libros I-X, y XX-XLV, que tratan el período de tiempo que abarca desde la fundación de Roma hasta la Segunda Guerra Púnica (218-201 a. C.).

10 Torrego 2007, p. 229, distingue la «circunstancia» o «circunstancia concomitante» de otras funciones, que «expresan una situación que enmarca la de la predicación a la que se adjunta». El «ablativo absoluto» desarrolla esta función, pues presenta un evento que circunscribe el que se recoge en la oración principal. 
racterísticas sintácticas (\$3.1.1) y semánticas (§3.1.2) que apuntan a un uso presentativo y posteriormente $(\$ 3.2)$ se analiza su valor en este nivel. El siguiente epígrafe (\$4) se dedica al «nivel interactivo», donde se estudia la motivación pragmática de forte $(\$ 4.1)$ y su valor epistémico $(\S 4.2)$. Tras el análisis de forte se ofrece en el capítulo $\$ 5$ un comentario de dos fragmentos problemáticos en Livio. Por último, en $\$ 6$ se ofrecen las conclusiones de este trabajo.

\section{NIVEL REPRESENTATIVO}

\subsection{Morfología y designación}

Forte es la forma en ablativo singular del sustantivo defectivo fors, declinable solo en los casos nominativo y ablativo ${ }^{11}$. Por su etimología se relaciona con fortuna, -ae, de similar designación, cuyo empleo es mucho más frecuente que fors en la literatura latina. Fors remite a una entidad definida como «fuerza natural o divinidad antropomórfica» que produce en el mundo determinados hechos de manera espontánea e imprevisible como causa indeterminada o casualidad, y está opuesto a otros términos que designan entidades de conducta o carácter ordenado y planificado, como es el caso de fatum 'hado', necessitas 'necesidad, ineludibilidad' y consilium 'planificación, determinación':

(5) sed mihi haec et talia audienti in incerto iudicio est, fatone res mortalium et necessitate immutabili, an forte uoluantur («Pero, cuando oigo este tipo de cosas, me resulta incierto si los asuntos de los mortales dependen del hado y de una necesidad inmutable o bien del azar», Tac. Ann. 6.22.1).

(6) consul alter, seu forte seu consilio nulli fugientium insertus agmini, cum quinquaginta fere equitibus Venusiam perfugit («El otro cónsul, que por casualidad o intencionadamente no se había incorporado en ninguno de los ejércitos que huían, huyó a Venusia con casi cincuenta caballeros», Liv. 22.49.14).

En época de Livio, la categoría gramatical de fors, al igual que forte, no está del todo definida ${ }^{12}$ :

(7) et fors aequatis cepissent praemia rostris ( $\mathrm{Y}$ acaso, igualados los espolones, se habrían hecho con los galardones», Verg. Aen. 5.232).

\footnotetext{
${ }^{11}$ Para la etimología de forte cf. DELL: s.v. Se documenta también la forma en genitivo fortis en la fórmula Fors Fortuna (Liv. 10.46.14 y 27.11.3).

${ }^{12}$ LTL s.v.: unde etiam fors et forte adverbii modo usurpantur («De donde también se utilizan fors y forte como adverbios»); DELL s.v.: «Fors» s'emploie adverbialement, au nominatif absolu formant une sorte de phrase nominale.
} 
Como se observa, en (7) fors no desempeña ninguno de los papeles semántico-sintácticos propios del sintagma nominal en nominativo (cf. Cabrillana 2007), entre los que destacan como prototípicas las funciones de sujeto, complemento del sujeto y aposición, sino que su alcance abarca el evento contenido en la oración (praemia capere 'hacerse con los galardones'), característica propia de los «satélites» o constituyentes opcionales de la predicación que se expresan generalmente en latín por medio de adverbios, sintagmas preposicionales y sintagmas nominales en ablativo, no a través del nominativo.

Si fors se encuentra en un estadio intermedio entre la categoría de sustantivo y la de adverbio, con más razón lo estará forte, cuya forma en ablativo expresa una noción en muchos casos cercana a la de un adverbio. Sin embargo, su distinta interpretación tendrá efectos directamente sobre la semántica de esta forma, pues forte como sustantivo en ablativo refiere una entidad que produce hechos en el mundo (la fortuna que actúa), mientras que como adverbio es más bien una propiedad, la manera en la que la situación se desarrolla ${ }^{13}$. En el caso de Livio la categoría morfológica de forte, por norma general, no está determinada, sino que depende de la interpretación del lector. Sin embargo, no faltan casos como los de (8), donde forte se presenta como un claro sustantivo, capaz de actuar como núcleo de un sintagma nominal:

(8) forte quadam utili ad tempus («Por una cierta casualidad útil a las circunstancias», Liv. 3.64.4).

En (8) forte se manifiesta como un sustantivo completo, con rección nominal, refiriendo una entidad, bien una divinidad, bien una fuerza de la naturaleza que provoca el evento señalado. En los demás casos, en los que no hay indicio alguno sobre la categoría de forte, queda a discreción del lector si es un sustantivo que refiere una entidad o un adverbio que expresa una propiedad.

\subsection{Características sintácticas}

En Livio forte es un elemento periférico. En efecto, en la estructura oracional se presenta en la totalidad de los casos como «satélite» de la predicación, afectando al conjunto formado por el predicado y sus

\footnotetext{
${ }^{13}$ Dik 1997, p. 136 y ss., a partir de Lyons 1977, distingue cinco tipos de entidades: la «propiedad» (property/relation), la «entidad espacial» (spatial entity), el «estado de cosas» o «evento» (state of affairs), el «hecho posible» (possible fact) y el «acto de habla» (speech act). En este trabajo simplificamos las opciones en dos categorías semánticas básicas, «entidad»y «propiedad», distintas entre sí según su autonomía -o no- existencial: la «entidad» posee existencia autónoma; la «propiedad» necesita adscribirse a una «entidad» para su realidad.
} 
argumentos. Esta integración se constata a través de su coordinación con otros elementos típicamente periféricos ${ }^{14}$, $\operatorname{como}$ es el caso de los constituyentes de expresión de «causa», «satélites» de la «predicación expandida ${ }^{15}$ :

(9) atque illa simulationum nescia, cum propter discumberet, non uultu aut sermone flecti, nullos attingere cibos, donec aduertit Tiberius, forte an quia audiuerat ( YY ella, inexperta en la disimulación, al recostarse cerca, no cambió su rostro ni su diálogo, no tocó alimento alguno, hasta que Tiberio lo advirtió, por casualidad o porque se lo habían dicho», Tac. Ann. 4.54.1).

(10) id acciderit ob iniquitatem praepotentium an forte, non satis constat ( $\mathrm{Si}$ esto sucedió por la iniquidad de los líderes o por casualidad, no está lo suficientemente claro», Plin. Nat. 12.93).

En el corpus no encontramos ningún caso en el que forte sea un elemento obligatorio, lo que no sorprende, si consideramos que la forma en ablativo es marca típica de constituyentes periféricos y solo en casos excepcionales argumento. Una búsqueda más amplia en autores latinos de época clásica a través de la base de datos BTL2 no ha ofrecido tampoco ningún caso en el que forte sea argumento, aunque no se descarta que eventualmente pueda desarrollar este papel en alguna estructura predicativa con régimen en ablativo.

Otra característica sintáctica de forte es su preferencia por las primeras posiciones de la oración, alejado del predicado al que se refiere, lo que se observa con claridad en períodos de mayor extensión y complejidad:

(11) forte ea regione, qua M. Sergius tribunus militum praeerat, castra adorti sunt ingentemque terrorem intulere, quia Etruriam omnem excitam sedibus magna mole adesse Romani crediderant ( «Casualmente por esta región, en la que estaba al frente el tribuno militar Marco Sergio, atacaron el campamento y trajeron gran terror, pues creían que toda Etruria, saliendo de sus territorios, acudía con una gran mole», Liv. 1.5.6).

Comparte esta predilección con otros constituyentes oracionales periféricos que sirven como escenario de los eventos, normalmente situados al principio del período que circunscriben:

\footnotetext{
${ }^{14}$ Dik 1968 señala que la coordinación de dos constituyentes requiere que su nivel de integración sea el mismo.

${ }_{15}$ Dik 1997, p. 77, distingue tres niveles en la predicación: la «predicación nuclear» (nuclear predication), la "predicación básica» (core predication) y, la más periférica, la "predicación extendida» (extended predication). La «causa» en latín se puede expresar a través del ablativo (cf. Torrego 2009), construcciones preposicionales (cf. Baños 2009), adverbios (Tarriño 2009a) u oraciones subordinadas (Tarriño 2009b).
} 
(12) et tum forte certamen ad portam erat ( $\mathrm{Y}$ casualmente entonces el enfrentamiento se estaba produciendo ante la puerta», Liv. 10.42.2).

(13) per eosdem forte dies et Bomilcar cum militibus ad supplementum Carthagine missis elephantisque et commeatu Locros accessit («Casualmente, por aquellos días también Bomílcar acudió a Locros con los soldados, elefantes y equipaje que habían sido enviados como apoyo desde Cartago», Liv. 1.24.1).

(14) forte, epulantibus iis, cum iam uino incaluissent, nuntiatum... («Casualmente, mientras cenaban, cuando ya entraron en calor con el vino, se anunció que...», Liv. 9.42.10).

En los tres casos forte aparece junto a constituyentes circunstanciales que sitúan el evento al que se refieren. En (12) aparece junto a tum y en (13) junto a per eosdem dies, ambos indicando simultaneidad temporal. En (14) forte viene acompañado de un «ablativo absoluto», que introduce un evento que sirve como espacio en el que se desarrolla otro evento principal ${ }^{16}$.

\subsection{Características semánticas}

\subsubsection{Función semántica: Causa y Modo}

Los estudios y diccionarios coinciden en que forte sirve como expresión de las funciones Causa y Modo. Determinados casos en los que aparece coordinado con otros constituyentes que desempeñan estas funciones semánticas lo corroboran, pues un constituyente solo puede coordinarse con otros elementos del mismo nivel de integración y misma función semántica ${ }^{17}$. En los ejemplos anteriores de (9) y (10) forte se muestra como la Causa, pues en un caso se coordina con una oración introducida por el nexo causal quia (9) y en otro con el sintagma $o b+$ acusativo (10), que también codifica en latín dicha función ${ }^{18}$. Existen también ejemplos en los que forte aparece coordinado con otros elementos de expresión de modo, como raptim:

(15) nec quicquam raptim aut forte temere egeritis ( $Y$ no hagáis nada a escondidas o por casualidad temerariamente», Liv. 23.3.3).

Cuando forte se interpreta como Causa remite a una entidad concreta o abstracta que provoca unos hechos en el mundo representado y,

\footnotetext{
16 Cf. Baños 1994.

17 Cf. Dik 1968 y Villa 1989.

${ }^{18}$ Cf. Torrego y Villa 2009, p. 69.
} 
como Modo, presenta una propiedad de un evento. El límite entre la Causa y el Modo, entre forte como entidad o como propiedad, no es siempre clara, un hecho que se manifiesta en las traducciones. Presentamos a continuación dos extractos de la traducción de Villar 1990:

(16) forte ita incidit, ut in Ser. Sulpici tribuni militum domo sorores Fabiae cum inter se, ut fit, sermonibus tempus tererent, lictor Sulpici, cum is de foro se domum reciperet, forem, ut mos est, uirga percuteret ( Quiso la casualidad que, estando en casa de Servio Sulpicio, tribuno militar, las hermanas Fabias pasando el rato charlando como es corriente, el lictor de Sulpicio, cuando se retiraba del foro a su casa, golpeó la puerta con la vara según la costumbre», Liv. 6.34.6).

quod ubi animaduertit Camillus, ignem in obiectam saepem conici iussit, et forte erat uis magna uenti uersa in hostem ( CCuando Camilo lo advirtió, dio orden de prender fuego al cercado que les cerraba el paso; casualmente el viento soplaba con gran fuerza en dirección al enemigo", Liv. 6.2.10).

En (16) y (17) se ofrecen las dos lecturas posibles de forte. En (16) actúa como una entidad que de alguna manera es responsable del evento: actúa, participa e incluso «quiere». Por otra parte, en (17) Villar opta por traducirlo como una propiedad del evento a través del adverbio «casualmente». Esta ambigüedad no sorprende, si consideramos que el ablativo es una marca capaz de desarrollar múltiples funciones ${ }^{19}$.

\subsubsection{Forte y el parámetro de «control»}

Forte, sea Modo o Causa, ejerce cierto influjo sobre el parámetro semántico de control de la predicación en la que aparece ${ }^{20}$. En primer lugar, recordemos que como expresión de Causa forte es la entidad «instigadora $»^{21}$ del evento en el que se halla o, dicho de otra manera, la fuente a partir de la cual fluye la situación. En los eventos en los que aparece con esta función se pueden percibir dos momentos de «instigación»: un nivel más nuclear, donde un participante (p. ej. un Agente

\footnotetext{
${ }^{19}$ El ablativo puede expresar Lugar, Tiempo, Instrumento, Compañía, Causa, Modo, Circunstancia, Precio, etc., (cf. Torrego 2009).

${ }^{20}$ Un evento es «controlado» cuando uno de los participantes centrales, el Agente, tiene control sobre el evento. Esta propiedad se relaciona con la «intencionalidad» $\mathrm{y}$ «voluntad» de provocar un determinado «estado de cosas» y, por lo tanto, es exclusiva del Agente y ausente en eventos en los que el «instigador» coincide con una entidad sin el rasgo de «intención» ni «voluntad», como corresponde a la Fuerza, Instrumento y Causa (cf. Dik 1997, p. 112).

${ }^{21}$ En Naess 2007 se define la función de instigation como la capacidad de provocar una determinada situación, un rasgo característico de las funciones de Agente, Causa y Fuerza, que vienen desarrolladas por entidades que -de alguna manera- se presentan como responsables de que un determinado estado de cosas se dé en el mundo representado.
} 
o Fuerza ${ }^{22}$ ) es el responsable directo de la situación, y otro más periférico, en el que otro participante (p. ej. una causa o motivo ${ }^{23}$ ) provoca de manera indirecta que el primer participante actúe:

(18) nam forte quadam, priusquam infanda merces perficeretur, per altercationem nondum omni auro appenso dictator interuenit auferrique aurum de medio et Gallos summoueri iubet ( «En efecto, por cierta casualidad, antes de que se llevara a término el infando comercio, a través de un altercado, cuando todavía no se había pesado todo el oro, el dictador interviene y ordena quitar de en medio el oro y llevarse a los galos», Liv. 5.49.1).

En el ejemplo (18) el contenido nuclear es la intervención (intervenire) del dictador (dictator) y, en un nivel más externo, es el azar el que, después de todo, da al dictador la oportunidad de actuar, situándolo en el lugar y momento oportunos. Hay por tanto dos planos de actuación diferente en el mismo evento, la del dictador y la de forte, cada una de ellas con su propio rasgo de control: el dictador, como humano con consciencia y voluntad, actúa en este caso con control; forte, por el contrario, actuará con control si se interpreta como una divinidad con consciencia e intención, o sin control si se considera que remite a una ley natural sin voluntad ni intencionalidad. Resulta en definitiva que, dado que el evento encuentra su origen último en la actuación de forte, el control del dictador no es absoluto. De aquí que los eventos controlados en los que aparece forte se presenten como imprevistos, espontáneos, azarosos y, en definitiva, no del todo controlados por los participantes agentivos.

Por otro lado, en los casos en los que forte se presenta, no como entidad causante o Causa, sino como propiedad del evento o Modo, se entiende que el evento se produce de manera espontánea y no planificada, cuyas consecuencias el Agente no ha previsto ni medido, lo que dota al evento del rasgo de «no controlado». El efecto es similar a lo que hemos considerado en el caso de forte como expresión de Causa, con la diferencia de que ahora no se considera una entidad que actúa en un plano superior, sino que se da cuenta sencillamente del modo no planificado en el que se actúa. Cuando forte como Modo se añade a predicados «controlados» expresa que el Agente que realiza la acción, en principio controlada e intencionada, no es consciente del alcance general de la misma, cuyas consecuencias pueden ser, como en (19), negativas:

(19) ex equo tum forte Mettius pugnabat; eo pelli facilius fuit («Casualmente, Mecio estaba luchando a caballo. Por ello fue más fácil expulsarlo», Liv. 1.12.9).

\footnotetext{
${ }^{22}$ La Fuerza se define en Dik 1997, pp. 118-119, como una entidad que provoca una determinada situación sin voluntad ni control.

${ }^{23}$ En Torrego 2009 el Motivo se define como una Causa que da origen a las actuaciones humanas.
} 
En (19) Mettius pugnabat 'Mecio estaba luchando' es un evento, en principio, controlado: Mecio lucha sabiendo lo que hace y con intención clara de hacer lo que hace. Forte indica, sin embargo, que esta acción se produce de manera espontánea y no planificada, pues si evidentemente Mecio fuera consciente de que su lucha a caballo permitiría a los enemigos expulsarlo con mayor facilidad, lo más seguro es que buscara otro modo de guerrear. De este modo, una acción que en principio parece «controlada» por un participante humano agentivo se presenta hasta cierto punto como «incontrolada», o no del todo «controlada», por medio de forte.

El efecto de forte en el parámetro de «control» lo hace compatible con otras formas de expresar la imprevisión, espontaneidad y descontrol. Este es el caso de forte temere, estructura prácticamente fosilizada, presente en Livio $^{24}$ :

(20) una uox audiebatur ad mare uocantium; id forte temere ab uno exclamatum totis passim personabat castris ( Se oía solo la voz de los que llamaban al mar. Lo que por casualidad había sido exclamado temerariamente por un solo individuo resonaba por todas partes en el campamento», Liv. 41.2.7).

Una de las cuestiones que plantea forte temere es si actúa como unidad funcional o si, por el contrario, cada elemento conserva cierta independencia. En (20) parece que ambos pueden interpretarse con un valor ligeramente distinto: mientras que temere expresa un modo de actuar temerario e irreflexivo, forte parece presentar el hecho de manera casual, espontánea o imprevista. La locución forte temere merece, sin embargo, un estudio más amplio y específico de su significado y función en un corpus diacrónico, pues frecuentemente alterna con forte ac temere. Esta situación plantea, primero, la cuestión de si es un estadio anterior o posterior a forte temere y, por otro lado, si forte posee o no una función y significado similar a temere.

\section{NiVEL PRESENTATIVO}

\subsection{Orientación presentativa de forte}

\subsubsection{Comportamiento sintáctico}

En el corpus analizado forte presenta un comportamiento sintáctico que coincide con el de otras partículas y constituyentes oracionales usados en la organización de los eventos en una narración. En los datos

${ }^{24}$ En Livio aparece 6 veces, un 3\% de los casos. En la base de datos BTL2 encontramos 13 casos, 10 de forte temere y 3 de forte ac temere, casi un $1 \%$ de los casos. 
se observa que forte tiende a aparecer al principio de la oración, en un nivel bastante periférico y alejado, por tanto, del predicado:

(21) forte postero die quam Subota classis tenuit quinque et triginta naues, quos hippagogus uocant, ab Elaea profectae cum equitibus Gallis equisque, Phanas, promunturium Chiorum, petebant, unde transmittere in Macedoniam possent; Attalo ab Eumene mittebantur («Casualmente, un día después de que la flota hubiera fondeado en Subota, treinta y cinco naves, que llaman hipagogos, que habían salido de Elea con jinetes galos y caballos, se dirigían a Fanas, al promontorio de Quíos, desde donde se podría pasar a Macedonia», Liv. 44.28.7).

Estas características se manifiestan también en otros elementos de marcación discursiva, cuya posición inicial les permite, como engranajes, conectar la oración en la que aparecen con lo dicho anteriormente, estableciendo unas relaciones semánticas determinadas que afectan, no a los constituyentes oracionales en particular, sino a los contenidos representados en general ${ }^{25}$. Así, los adverbios y otras formas de expresión temporal poseen una clara función presentativa, pues organizan en la línea temporal (en la narración) o espacial (en la descripción) los contenidos representados ${ }^{26}$. En (22) el sintagma per eos dies "por aquellos días' permite al lector organizar los hechos narrativos en la línea temporal, no como eventos posteriores a lo dicho, sino como coincidentes con el período de tiempo notado:

(22) per eos dies consul Nautius ad Eretum cum Sabinis egregie pugnat ( $\mathrm{Por}$ aquellos días el cónsul Naucio lucha magníficamente con los Sabinos en Ereto», Liv. 3.29.7).

Otro indicio más del valor presentativo de forte es que, como otras partículas y adverbios presentativos, puede aparecer en posición tmética en medio de un sintagma al que no pertenece, propiedad frecuente en adverbios y partículas presentativas (igitur, tamen, enim, etc.) y otros adverbios de expresión circunstancial, especialmente de ubicación espacio-temporal, cuyo valor en la organización de contenidos está ya demostrada. En (23) y (24) observamos un comportamiento similar entre forte y el adverbio temporal tum 'entonces'.

(23) eo forte tempore Camillus cum exercitu Romano interuenit («Casualmente por aquel tiempo Camilo llegó con su ejército», Liv. 6.3.3).

(24) hoc tum modo ab Latinis repetitae res ac bellum indictum ( Entonces, de este modo, los latinos retomaron el asunto y declararon la guerra», Liv. 1.32.14).

${ }^{25}$ Cf. Aijmer 2002, pp. 28-30.

${ }^{26}$ Cf. el valor de nunc en Risselada 1996. Sobre la polifuncionalidad de los constituyentes de expresión circunstancial, cf. Aijmer 2002, p. 27. 


\subsubsection{Características semánticas}

Hemos visto que el comportamiento sintáctico de forte coincide con el de otros adverbios y sintagmas de expresión local, temporal o circunstancial empleados en la organización de los contenidos narrativos. Estos establecen el fondo circunstancial de la predicación, información que pone en relación el evento con lo dicho anteriormente. Forte aparece frecuentemente junto a estos constituyentes: en (25) se yuxtapone a un Satélite de Tiempo (tum 'entonces'), en (26) a un Satélite de Lugar (in duobus exercitibus 'en los dos ejércitos') y en (27) aparece junto a un Satélite que expresa la Circunstancia concomitante ${ }^{27}$ (epulantibus iis, cum iam vino incaluissent 'cenando estos, cuando ya con el vino habían entrado en calor').

(25) et tum forte certamen ad portam erat («Y entonces casualmente el combate se producía ante la puerta», Liv. 10.42.2).

(26) forte in duobus tum exercitibus erant trigemini fratres nec aetate nec uiribus dispares («Casualmente en los dos ejércitos había entonces tres hermanos trillizos, parejos en edad y fuerza», Liv. 1.24.1).

(27) forte, epulantibus iis, cum iam uino incaluissent, nuntiatum in conuiuio esse nobilem Boium cum liberis transfugam uenisse («Casualmente, cenando estos, cuando ya con el vino habían entrado en calor, se anunció en el convite que un noble boyo fugitivo había venido con sus hijos», Liv. 39.42.10).

Estos constituyentes circunstanciales (tiempo, espacio, modo, etc.), incluido forte, son un instrumento fundamental para construir y ordenar los distintos episodios narrativos, entendiendo por «episodio» una unidad semántica que contiene un conjunto de eventos que comparten un mismo fondo común (ubicación espacio-temporal, participantes, etc. ${ }^{28}$. Por ejemplo, los adverbios postea 'después', antea 'antes', interim 'mientras tanto' y los sintagmas in eo tempore 'en aquel momento' o per eos dies 'por aquellos días' establecen una relación temporal de posterioridad, anterioridad o simultaneidad entre dos contenidos eventivos, al igual que los constituyentes locales, del tipo eo loco 'en aquel lugar' o $i b i$ 'allî', que presentan también continuidad o discontinuidad narrativa desde el plano espacial. A estos parámetros hay que añadir también la introducción o salida de escena de los personajes de la narración, que sin duda contribuyen en la organización de los contenidos narrativos.

\footnotetext{
${ }_{27}$ Sobre esta función cf. Torrego 2009: «La función Circunstancia o Circunstancia concomitante expresa una situación que enmarca la de la predicación a la que se adjunta».

${ }^{28}$ Cf. Hengeveld y Mackenzie 2008, pp. 157-166.
} 
Pues bien, los datos del corpus muestran que forte aparece frecuentemente en contextos de cambio narrativo. En (28), por ejemplo, aparece en una oración en la que se introduce un nuevo personaje, Timasiteo, en un período de tiempo que coincide con los eventos acaecidos anteriormente, lo que se indica a través de la expresión temporal eo anno 'ese año'. El fondo circunstancial expresado por esta estructura y el nuevo personaje son suficientes para considerar que se trata de un nuevo «episodio» en la narración, una unidad semántica con un fondo situacional común ${ }^{29}$ :

(28) forte eo anno in summo magistratu erat Timasitheus quidam, Romanis vir similior quam suis, qui... («Casualmente ese año en la suma magistratura estaba un cierto Timasiteo, un hombre más parecido a los romanos que a los suyos, el cual...», Liv. 5.28.3).

Por otra parte, en (29) se presentan las circunstancias de la escena en la que los agentes del rey Amulio abandonan en el río Tíber a los gemelos Rómulo y Remo:

(29) forte quadam diuinitus, super ripas Tiberis effusus lenibus stagnis nec adiri usquam ad iusti cursum poterat amnis et posse quamuis languida mergi aqua infantes spem ferentibus dabat ( Por cierta casualidad divina, el Tíber, que se había desbordado, no permitía acceder hasta el cauce normal debido a los lentos estancamientos, y daba la esperanza a los que llevaban a los infantes de que se sumergieran en el agua a pesar de su tranquilidad», Liv. 1.4.4).

Forte puede, en algunos casos, aparecer como único índice de cambio circunstancial en la narración, como en (30), donde no se observa cambio temporal, local ni de participantes:

(30) forte ea cura, quae aduersus Poenos praeparata erat, aduersus Siculos usui fuit («Casualmente, las medidas que habían tomado contra los púnicos les sirvieron contra los sículos», Liv. 24.36.1).

En definitiva se observa que forte posee un valor en la organización de contenidos narrativos a partir de dos indicios: primero, por su posición al inicio de nuevas cadenas de eventos y, segundo, por su comportamiento cercano al de otros elementos que actúan en el «nivel presentativo».

\subsection{Caracterización de la función presentativa de forte}

En $§ 2.1$ se dijo que forte, relacionado con fortuna, designa un ente divino o fuerza natural abstracta e indeterminada que produce de-

\footnotetext{
${ }^{29}$ Cf. nota 28.
} 
terminados hechos en el mundo, lo que hace que se manifiesten en muchos casos en los eventos, bien como la entidad causante de la situación representada, bien como la propiedad o modo en el que se produce $(\$ 2.3 .1)$.

La sintaxis y semántica de forte apuntan a un uso especial del mismo en la narración, no como mero «satélite» opcional, sino más bien como mecanismo dedicado a la ilación de eventos desde un punto de vista secuencial-causal. Forte establece, en efecto, una relación de «discontinuidad episódica ${ }^{30}$. Por medio de este adverbio el hablante señala que existe una discontinuidad secuencial de tipo causal entre el evento precedente y aquel en el que aparece o, dicho de otra manera, forte presenta un evento independiente, no provocado por los precedentes. Como «causa», el evento se presenta originado por una divinidad caprichosa o fuerza de la naturaleza impredecible; como «modo», el evento se presenta como casual, espontáneo, indeterminado, no planificado, sin motivo o causa conocida. En este sentido forte se opondría a aquellas expresiones causales que señalan que un evento se produce a partir de otro, del tipo unde 'de donde', quare 'por esta causa' o ideo 'por ello', que indican una continuidad causal transitiva en la cadena de eventos ${ }^{31}$.

El valor presentativo de forte no se manifiesta solo entre los contenidos eventivos de oraciones principales (31), sino que actúa también en un nivel más local, indicando que el evento contenido en una oración subordinada no encuentra su origen en el evento de la oración principal (32):

(31) per eosdem forte dies M. Iunius consul ex Histria comitiorum habendorum causa Romam uenit («Casualmente por aquellos días el cónsul Marco Junio vino a Roma desde Histria para celebrar los comicios», Liv. 41.7.4).

(32) dum haec loquuntur, iumenta forte pascentia extra uallum Gallo abigenti duo milites Romani ademerunt («Mientras hablan estas cosas, dos soldados romanos robaron unos jumentos pastoreados por un galo, los que por casualidad estaban pastando fuera de la empalizada», Liv. 7. 14.4).

En (31) la llegada a Roma del cónsul Junio en ese determinado período se presenta como un evento independiente de lo dicho anteriormente. En (32) la escena de los jumentos pastando fuera de las vallas se presenta también como un hecho casual, cuyas causas profundas se desconocen.

\footnotetext{
${ }^{30}$ Cf. Hengeveld y Mackenzie 2008, pp. 157 y ss.

${ }^{31}$ Este tipo de partículas presentan un evento $(e)_{x}$ causante de otro $(e)_{y}$, según la sucesión $\left[(\mathrm{e})_{1}>(\mathrm{e})_{2}>\ldots>(\mathrm{e})_{\mathrm{n}}\right]$. Forte rompe dicha sucesión y permite comenzar un orden distinto causal: $\left[\left[(\mathrm{e})_{1}>(\mathrm{e})_{2}\right] / /\left[\right.\right.$ forte $\left.\left.(\mathrm{e})_{3}>(\mathrm{e})_{4}>\ldots>(\mathrm{e})_{\mathrm{n}}\right]\right]$.
} 


\section{NiVEL INTERACTIVO}

\subsection{La motivación pragmática de forte}

A lo largo del epígrafe anterior (\$3.2) habíamos visto que, a menos que forte se integre en un contexto en el que se haga clara referencia a la Fortuna, por norma general el hablante no emplea esta forma para indicar una actuación relevante de dicha entidad en los eventos, sino más bien el modo espontáneo e imprevisible en el que se desarrolla. Este forte desdibujado y casi gramaticalizado es empleado por el hablante, bien para expresar que desconoce las circunstancias que provocan una determinada situación, bien para evitar dar explicaciones, posiblemente vanas o irrelevantes, sobre lo que produce un determinado acontecimiento.

En el primer caso el hablante acepta un evento determinado como real, pero se reconoce incapaz de traer a colación las causas de dicha situación. En el segundo caso el hablante evita dar cuenta de unos orígenes que decide no expresar, tal vez porque requieran un largo tratamiento, quizá porque sean tediosos, bien porque desvíen la trama hacia un asunto irrelevante. Mediante forte el hablante da cierta idea a su oyente sobre su disposición subjetiva en relación con los contenidos narrados, que el mismo hablante selecciona y presenta intentando que el acto comunicativo sea lo más eficaz posible.

\subsection{Forte y la modalidad epistémica}

En diccionarios y otros trabajos se habla de un forte de duda o probabilidad, en algunos casos con un matiz de ironía. Según estos estudios, el hablante transmite mediante forte su opinión respecto al evento que comunica, si lo considera probable, dudoso, imposible, etcétera.

Los datos no respaldan, sin embargo, este tipo de explicación. Ciertamente, forte aparece frecuentemente en contextos de expresión de duda o probabilidad, pero su comportamiento es muy diferente al de adverbios como, por ejemplo, fortasse 'quizá', que, en contextos similares, no desarrolla la misma función:

(33) erunt etiam fortasse, iudices, qui... («Habrá también quizá, jueces, quienes...», Cic. Verr. 1.11.68).

(34) forte eo anno in summo magistratu erat Timasitheus quidam... («Casualmente aquel año en la suma magistratura estaba un tal Timasiteo...», Liv. 5.28.3).

En ambos casos, en (33) y (34) el predicado verbal sum tiene el valor existencial equivalente al esp. «hay». En (33) el evento erunt qui... 
'habrá quienes' aparece como dudoso, en una oración mediante la cual el orador expresa la probabilidad de que la situación representada pueda producirse. Sin embargo, no cabe la misma interpretación en (34), donde erat Timasitheus quidam 'había un tal Timasiteo' se presenta como un evento cuya realidad el hablante no cuestiona.

Los casos de los que se valen los diccionarios y trabajos para ilustrar la expresión de duda del hablante a través de forte presentan distintos tipos de estructuras hipotéticas: condicionales $(s i, n i$, nisi), finales (ut, ne) e interrogativas (an). Forte no parece ser aquí la fuente de la hipoteticidad, que se expresa por medio de otros elementos:

(35) te id prius scire uolui, si forte abesse, dum facinus patratur, malles ( Quise que tú lo supieras primero, por si acaso hubieras preferido estar ausente mientras se llevaba a cabo el trabajo», Liv. 23.8.11).

(36) cum staret tacitus et circa eum aliquot hominum, ne forte uiolaretur, constitisset globus... ( Mientras permanecía callado y alrededor de él se situaba un grupo de hombres para que por casualidad no se viera atacado...», Liv. 2.29.2).

(37) quid enim prius Minerua perdiderat, ut periret? an forte custodes suos? ("¿Pues qué había perdido Minerva antes, para perecer? ¿Acaso sus guardianes?», August. C.D. 1.2) ${ }^{32}$.

En (35) el nexo condicional si es el marcador de hipoteticidad, en (36) el nexo final ne y en (37) la partícula interrogativa an. Si se elidiera forte de los ejemplos de (35)-(37), las oraciones no perderían su carácter hipotético, lo que es prueba concluyente de que este uso le es ajeno.

El comportamiento de forte en este tipo de contextos responde, en el «nivel representativo», al que hemos expuesto en $\$ 2.3 .1$ y $\$ 2.3 .2$, como expresión de Causa o Modo que afecta la propiedad de «control» del evento en el que se integra. La expresión de una causa tan externa y desdibujada o de un modo espontáneo e imprevisto, así como su posición junto a la forma hipotética pueden ser los motivos por los que se haya identificado forte con la duda más que como un elemento compatible con esta. En efecto, un evento se define como hipotético a través de distintos mecanismos gramaticales, sean nexos condicionales, finales o interrogativos. El hablante añade forte para notar que, de producirse el evento, sería imprevisto e inesperado, sorprendente y de aquí que pueda expresar que la posibilidad es, según su perspectiva, bastante remota. Forte no vuelve hipotético un evento, sino que afecta de alguna manera al carácter hipotético ya presente.

Comparando la secuencia si forte con el mero nexo condicional si se observa la siguiente diferencia: mientras que si introduce una situa-

\footnotetext{
${ }^{32}$ Ejemplo en Orlandini 1997.
} 
ción posible, si forte, además de esto, indica que evento referido sería casual e inesperado, más díficil de producirse, con menos posibilidades de realizarse:

(38) an est quisquam, qui dubitet nullis iniuriis vestris, si quae forte aliquando fuerunt, $[\ldots]$ tribunos plebis offensos ac concitatos esse? («¿ O es que hay alguno que pueda dudar de que ninguna injuria vuestra, si es que por casualidad hubo alguna, en algún momento [...] haya ofendido y airado a los tribunos de la plebe?», Liv. 41.24.12).

(39) itaque introductus in senatum gratulatus uictoriam est sua merita eo bello fratris que, si qua erant, et Gallorum defectionem, quae nuper ingenti motu facta erat, exposuit ( «Así, cuando entró a formar parte del senado se congratuló de su victoria y expuso sus méritos en aquella guerra, también los de su hermano, si alguno había, y la revuelta de los galos, que se había producido recientemente con gran conmoción», Liv. 45.20.1).

En los ejemplos (38) y (39) la oración subordinada condicional contiene un evento existencial a través de una estructura con el predicado sum. Ambas oraciones son hipotéticas, a pesar de la ausencia de forte en si qua erant 'si alguno había' en (39). En la estructura condicional forte añade una información subjetiva sobre el evento referido, al que el hablante presenta como casual o inesperado. Esta consideración subjetiva del hablante presenta la hipotética como poco probable o incluso imposible, lo que hace que forte sea muy frecuente en contextos irónicos como el del siguiente ejemplo:

(40) nemo enim fere saltat sobrius, nisi forte insanit ( $«$ Pues nadie baila, por lo general, sobrio, a no ser que esté loco», Cic. Mur. 13).

Cicerón señala en (40) que, por norma general, uno baila cuando está ebrio, siendo, según es su opinión, un hecho casual e inesperado que un individuo dance movido por una especie de locura o frenesí. Aunque Cicerón no excluye la insania como origen del bailar, la presenta como una causa inesperada y muy poco probable.

\section{COMENTARIO DE FORTE EN DOS EPISODIOS NARRATIVOS DE AB URBE CONDITA}

Los diccionarios y trabajos dedicados a forte aluden a casos claros en los que esta forma desarrolla una función fácilmente aprehensible. Así, proponen, para el uso presentativo, aquellos casos en los que forte aparece a principio de capítulo, introduciendo nuevos contenidos narrativos o, para su uso causal, presentan contextos en los que forte hace clara referencia a la divinidad. En este apartado hemos querido va- 
lernos de dos pasajes de Livio, 1.7.11-13 y 5.5.7, donde la interpretación de forte no es tan clara. En ambos casos la forma no aparece al principio de una escena narrativa, sino en su parte central, aparentemente con un valor muy distinto: mientras que en 1.7.11-13 parece introducir una digresión en el relato, en 5.5.7 parece presentar dos estructuras paralelas.

\subsection{Un sacrificio en honor a Hércules (Liv. 1.7.11-13)}

El primer fragmento que proponemos procede de Ab urbe condita 1.7.11-13. En él se relata cómo Evandro, rey de los arcadios, por recomendación de Carmenta, su madre, recibe a Hércules en sus territorios y prepara un sacrificio favorable, preludio de su apoteosis. Durante el festejo acuden los invitados, entre ellos los miembros de dos importantes familias, los pinarios y los poticios. En estas circunstancias sucede que los poticios son puntuales a la cita, mientras que los pinarios se demoran y han que conformarse con los restos del banquete. A partir de este hecho se instituyó que la familia siguiera alimentándose de las sobras en el aniversario de la celebración, tradición que se mantuvo, según Livio, hasta que la familia se extinguió:

(41) dextra Hercules data accipere se omen impleturumque fata ara condita ac dicata ait. ibi tum primum boue eximia capta de grege sacrum Herculi, adhibitis ad ministerium dapemque Potitiis ac Pinariis, quae tum familiae maxime inclitae ea loca incolebant, factum. forte ita euenit ut Potitii ad tempus praesto essent iisque exta apponerentur, Pinarii extis adesis ad ceteram uenirent dapem. inde institutum mansit donec Pinarium genus fuit, ne extis eorum sollemnium uescerentur ( Tras dar su derecha Hércules, dijo que aceptaba la profecía y que obedecería a su destino fundando y dedicando el altar. Allí entonces se desarrolló el primer sacrificio a Hércules con un buey excelente extraído del rebaño, participando en el servicio y en el banquete los poticios y pinarios, familias muy famosas que en aquel momento habitaban estos lugares. Casualmente sucedió que los poticios llegaron a tiempo y se les sirvió las entrañas, y que los pinarios, una vez se retiraron las entrañas, llegaron al resto del banquete. De aquí se permaneció la tradición de que mientras existiese la estirpe de los pinarios no comieran de las entrañas de esta solemnidad», Liv. 1.7.11-13).

En (41) forte introduce en la narración una digresión, es decir, un conjunto de eventos interesantes según la opinión del hablante, pero que interrumpen por un tiempo la cadena narrativa de los eventos de primer plano, centrándose en otros.

Un análisis que considere los tres niveles del discurso notará, en primer lugar, que forte aparece en posición inicial absoluta en la oración con un alcance muy amplio. Efectivamente, forte se aplica al contenido 
del predicado euenit 'sucedió' y, por extensión, al de los dos eventos expletivos que lo siguen: la llegada pronta de los poticios y la dilación de los pinarios. Desde el punto de vista semántico, forte expresa indistintamente la «causa», bastante desdibujada, o el «modo» en el que se produce la situación representada, presentando los eventos como fortuitos, casuales, no planeados o espontáneos.

En la presentación de contenidos forte adquiere un papel determinante, pues al indicar que el origen de dichos eventos es indeterminado, desliga su existencia a la de los anteriores, confiriéndoles cierta autonomía y liberándolo de la cadena causal narrativa anterior. En efecto, la pronta asistencia de los poticios y el retraso de los pinarios se deben a motivos o causas no notadas por el historiador, pero, sin duda, independientes de los eventos expresados en las oraciones precedentes.

En el «nivel interactivo», el autor emplea forte para dar cuenta de la irrelevancia o desconocimiento de las causas reales de los eventos a los que refiere. De esta manera evita ofrecer demasiada información a su público y presenta así directamente los eventos que le interesan.

En conjunto, en este pasaje forte indica, en el «nivel representativo», que el evento se produce de un modo casual o por una causa indeterminada; en el «nivel presentativo», desliga los eventos referidos a lo dicho anteriormente, confiriéndoles autonomía como escena independiente; $y$, en el «nivel interactivo», permite omitir una información irrelevante. En definitiva, el valor digresivo de forte se interpreta como un cúmulo de funciones de distinto nivel que, en conjunto, permiten la introducción de este tipo de contenidos.

\subsection{La infancia de Rómulo y Remo (Liv. 5.5.7)}

El segundo episodio que analizaremos es el de 5.5.7, que corresponde con la infancia y juventud de Rómulo y Remo. El fragmento refiere un momento cronológicamente posterior a la captura y entrega de Rómulo a Númitor, su abuelo. Aquí Livio relata que Fáustulo, padre adoptivo de los gemelos, se ve obligado a descubrir su origen real a Remo, hermano del apresado Rómulo. En un momento coincidente Númitor, que mantiene en custodia a Rómulo, descubre por sí mismo este mismo hecho:

(42) iam inde ab initio Faustulo spes fuerat regiam stirpem apud se educari; nam et expositos iussu regis infantes sciebat et tempus quo ipse eos sustulisset ad id ipsum congruere; sed rem immaturam nisi aut per occasionem aut per necessitatem aperiri noluerat. necessitas prior uenit: ita metu subactus Romulo rem aperit. forte et Numitori cum in custodia Remum haberet audissetque geminos esse fratres, comparando et aetatem eorum et ipsam minime seruilem indolem, tetigerat animum memoria nepotum; sciscitandoque eodem peruenit ut haud 


\begin{abstract}
procul esset quin Remum agnosceret. ita undique regi dolus nectitur («Ya desde el principio Fáustulo tenía la esperanza de que en su casa estuviera creciendo una estirpe real; pues sabía tanto que los niños habían sido expuestos por mandato del rey como que el momento en el que él mismo los había acogido coincidía con aquel otro; pero no había querido dar a conocer un tema aún inmaduro, a no ser en una ocasión favorable o por necesidad. Primero vino la necesidad: movido por el miedo se lo descubrió a Rómulo. Casualmente también a Númitor, que tenía preso a Remo y había oído que los hermanos eran gemelos, le había venido a la mente el recuerdo de sus nietos al relacionar su edad y su carácter nada servil, e investigando llegó a un punto en que no estuvo lejos de reconocer a Remo. Así, por las dos partes se teje una trampa al rey», Liv. 5.5.7).
\end{abstract}

A diferencia de (41), en (42) forte no parece introducir un contenido nuevo, sino proseguir con la escena que se está representando. En este caso introduce un evento paralelo a otro que se produce en un momento coincidente, por lo que se podría interpretar que forte marca cierta concomitancia.

En el nivel representativo, forte parece volver a indicar el modo inesperado y espontáneo en el que se produce el evento. En efecto, a diferencia del caso de Remo, que descubre su origen real a través de la confesión de Fáustulo, Númitor lo descubre de manera fortuita, al haber oído por casualidad que su prisionero tenía un hermano gemelo. La posición inicial de forte, alejado del predicado en un período tan extenso muestra también un alcance amplio de la forma sobre el contenido eventivo subsiguiente. En el nivel presentativo forte marca la autonomía causal del evento en el que aparece, pues indica que no viene motivado por ninguno de los eventos expresados anteriormente. Por último, en el nivel interactivo el autor evita tener que dar cuenta, primero, de cómo llegó a oídos de Númitor que los hermanos eran gemelos y, segundo, del proceso mental por el que recordó el abandono de sus nietos en el Tíber.

El paralelismo y coincidencia de ambos eventos no parecen venir de la mano de forte, sino del tiempo relativo de la narración y de la estructura semántica del acto de anagnórisis. Es et 'también' el elemento que presenta el nuevo evento paralelo, con una redistribución de participantes en el acto de reconocimiento: Númitor, en vez de Rómulo, es el que descubre el secreto y la casualidad, en vez de Fáustulo, es la que provoca dicha situación. La elisión de forte avalaría esta interpretación, pues el paralelismo de ambos eventos sería, aun con su ausencia, patente. De nuevo, y como ya sucedía en contextos hipotéticos, forte no marca ningún paralelismo entre dos eventos, si bien es compatible con este tipo de situaciones contextuales indicando la casualidad de la coincidencia. 
El valor de forte en este caso es, por tanto, similar al de (41): presenta la causa o modo en el que se produce un evento, permite expresar una situación como independiente de lo dicho anteriormente y ofrece al autor la posibilidad de omitir información innecesaria. La función presentativa de forte se orienta de otra manera, pues no introduce una nueva escena narrativa, sino que, por el contrario, presenta un evento que, aunque es independiente de los anteriores según su origen causal, sirve para proseguir la trama narrativa principal.

\section{CONCLUSIÓN}

El análisis de forte en Ab urbe condita de Livio ofrece los siguientes resultados:

- Primero, en el nivel representativo forte, como adverbio o sustantivo, es un satélite oracional que se integra en la periferia de la oración, en posición generalmente inicial, alejado del predicado y con un amplio alcance, expresando, bien la Causa o entidad que provoca un evento, bien el Modo en el que se desarrolla. En ambos casos forte afecta a la propiedad de control del evento en el que aparece, al que presenta como imprevisto o espontáneo.

- Segundo, en el nivel presentativo forte se ofrece como instrumento válido para la organización de los eventos narrativos, como demuestra su sintaxis y semántica, cercana a la de otros adverbios, partículas y constituyentes con valor presentativo. Forte marca discontinuidad entre el evento al que se refiere y los anteriores, interrumpiendo la secuencia causal de una determinada cadena de eventos, lo que le permite servir como introductor, bien de nuevas cadenas de eventos que conforman en conjunto un nuevo episodio narrativo, bien de eventos desligados de los anteriores en términos de causalidad, pero que de alguna manera son relevantes para el desarrollo de la narración.

- En el nivel interactivo el hablante se vale de forte para evitar dar cuenta de los orígenes etiológicos de una situación (de sus causas o motivos), bien porque los desconozca, bien porque no considera relevante dar cuenta de ello. A pesar de que forte es frecuente en períodos hipotéticos condicionales (si, nisi, ni), finales $(u t, n e)$ e interrogativos (an), no expresa propiamente la duda del hablante, sino que dota a la proposición hipotética de un cariz de imprevisibilidad y casualidad, que lleva a la interpretación de la improbabilidad o imposibilidad. 


\section{REFERENCIAS BIBLIOGRÁFICAS}

AJjMER, K. (2002): English discourse particles, Ámsterdam-Filadelfia, John Benjamins.

BAÑos, J. M. (1994): «Ablativo absoluto versus cum histórico: su distribución sintáctica en César», Actas del VIII congreso español de estudios clásicos, Madrid, Ediciones Clásicas, pp. 411-418.

- (2009): «Preposiciones», Sintaxis del latín clásico, Madrid, Liceus, pp. 297-345.

- et al. (eds.) (2003): Praedicativa: complementación en griego y latín, Universidade de Santiago de Compostela, Servicio de publicacións e intercambio científico.

BTL2 = Bibliotheca Teubneriana Latina (recurso digital: versión 2.0, K.G. Saur-BrepolsCTLO).

Cabrillana Leal, C. (2007): «Nominativo y vocativo», en Baños, J. M. (ed.), Sintaxis del latín clásico, Madrid, Liceus, pp. 111-130.

Champeaux, J. (1967): «Forte chez Tite-Live», Revue des Études Latines 45, pp. 363388.

DELL = ERnOUt, A. y MeILlet, A. (2001): Dictionnaire étymologique de la langue latine, París, Klincksieck.

DIK, S. C. (1968): Coordination: Its implications for the theory of general linguistics, Ámsterdam, University of Amsterdam.

- (1997): The theory of functional grammar, Berlín-Nueva York, Mouton de Gruyter.

HallidaY, M. A. K. y HaSAN, R. (1976): Cohesion in English, Londres, Longman.

HAND, F. (1969): Tursellinus seu de particulis latinis comentarii, Ámsterdam, Adolf M. Hakkert.

Hengeveld, K. y Mackenzie, J. L. (2008): Functional discourse grammar: A typologically-based theory of language structure, Oxford-Nueva York, Oxford University Press.

Hilton, J. (1989): «Temporal connectors in the narrative discourse of Cicero», Actes du Ve colloque de linguistique latine. Louvain-la-Neuve/Borzée. 31 mars - 4 avril 1989, pp. 173-184.

Kroon, C. (1995): Discourse particles in Latin. A study of nam, enim, autem, uero and at, Ámsterdam, J. C. Gieben.

Lyons, J. (1980): Semántica, Barcelona, Teide.

$L T L=$ ForCELLINI, A. et al. (1864-1926): Lexicon totius latinitatis, Padua, Arnaldus Forni.

NAEss, A. (2007): Prototypical transitivity, Ámsterdam-Filadelfia, John Benjamins.

OLD = GLARE, P. G. W. (ed.) (1992): Oxford Latin dictionary, Londres, Clarendon Press.

OrLANDINI, A. (1997): «Adverbes d'énoncé et adverbs d'énonciation en latin: forte, fortasse», LALIES 17, pp. 251-260.

Pinkster, H. (1995): Sintaxis y semántica del latín, Madrid, Ediciones Clásicas.

RisseladA, R. (1996): «And now for something completely different? Temporal discourse markers», en Risselada, R. y Bolkestein, A. (eds.), On Latin. Linguistic and literary studies in honour of Harm Pinkster, Ámsterdam, J. C. Gieben, pp. 105-125. 
TARriño, E. (2009a): «Adverbios y partículas», en Baños, J. M. (ed.), Sintaxis del latín clásico, Madrid, Liceus, pp. 349-374.

- (2009b): «Temporales y Causales», en Baños, J. M. (ed.), Sintaxis del latín clásico, Madrid, Liceus, pp. 601-632.

$T L L=$ AA.VV., Thesaurus linguae latinae, Leipzig, B. G. Teubneri.

TORREGO, M. E. (2002): «Statim, paulatim, repente/subito: Scope and lexical aspect», en Bolkestein, A. M. (ed.), Theory and description in Latin linguistics. Selected papers from the $\mathrm{XI}^{\text {th }}$ international colloquium on Latin linguistics, Amsterdam, June 24-29, 2001, Ámsterdam, J. C. Gieben, pp. 379-392.

- (2005): «Grammar and pragmatics: The textual uses of repente and subito», Papers on Grammar 9, 2, pp. 763-773.

- (2009): «Ablativo», en Baños, J. M. (ed.), Sintaxis del latín clásico, Madrid, Liceus, pp. 211-249.

- et al. (eds.) (2007): Praedicativa II: esquemas de complementación verbal en griego antiguo y en latín, Universidad de Zaragoza, Prensas Universitarias de Zaragoza.

- y Villa Polo, J. (2009): «La oración independiente: concepto. Estructura, constituyentes y niveles. Tipos», en Baños, J. M. (ed.), Sintaxis del latín clásico, Madrid, Liceus, pp. 55-82.

URízAR, J. R. (2010): «Análisis comparativo de los adverbios latinos fortasse y forsitan como expresión de modalidad epistémica», Actas del XXXIX Simposio de la Sociedad española de lingüistica, Santiago de Compostela, 1-4 de febrero de 2010, (recurso digital. D. L.: C 3689-2010).

VILLA, J. (1989): «Las funciones de los elementos nominales: Criterios para su identificación y caracterización en griego y latín», Cuadernos de Filología Clásica 22, pp. 291-304.

Villar, J. A. (trad.) (1990): Tito Livio: Historia de Roma desde su fundación. Libros IV-VII, Madrid, Gredos. 
(06)-Art. Urizar Salinas. RSEL 42-2:(7)-Revista Aq. n 84 14/1/13 12:23 Página 180 